\title{
Visibilidade do trabalho do enfermeiro no contexto do modelo clínico de assistência
}

\author{
Visibility of nursing work in the context of the clinical model of health care \\ Visibilidad del trabajo del enfermero en el contexto del modelo clínico de atención
}

\author{
Lenice Dutra de Sousa'; Wilson Danilo Lunardi Filho"I ; Maira Buss Thofehrn ${ }^{\text {III }}$
}

\begin{abstract}
RESUMO: O estudo objetivou analisar a visibilidade do trabalho do enfermeiro com base no exercício da clínica. Trata-se de uma pesquisa qualitativa e exploratória, na forma de estudo de caso, executada em uma unidade de internação cirúrgica. Foi pesquisado um grupo de seis enfermeiros e os dados foram coletados por meio de observação não participante e entrevista em profundidade. O período de coleta ocorreu de outubro de 2012 a janeiro de 2013 e os dados foram tratados por meio da análise textual discursiva. Verificou-se que a clínica, como campo de conhecimentos, torna o trabalho do enfermeiro mais visível para os diferentes sujeitos envolvidos no processo de saúde. Para tanto, é necessário o reconhecimento de que esse profissional incorpora a clínica ao seu fazer. Assim, a clínica pode ser um instrumento que possibilita o reconhecimento e consequente visibilidade do trabalho do enfermeiro e contribui nas discussões, negociações e transformações.
\end{abstract}

Palavras-Chave: Competência clínica; enfermagem; trabalho; enfermagem cirúrgica.

\begin{abstract}
This qualitative, exploratory case study examined the visibility of nursing in clinical practice in a surgery unit. The unit of analysis was a group of six nurses, and data were collected through non-participant observation and in-depth interview. Data was collected from October 2012 to January 2013, and were analyzed using discursive textual analysis. It was apparent that, as a field of knowledge, clinical practice makes the nurse's work more visible to the various people involved in the healthcare process. Therefore, the fact that the nurses incorporate clinical practice into their work needs to be acknowledged. That way, clinical practice can be an instrument that enables nurses' work to be acknowledged and consequently gain visibility, contributing to discussions, negotiations and transformations.

Keywords: Clinical competence; nursing; work; perioperative nursing.

RESUMEN: El estudio tuvo como objetivo analizar la visibilidad del trabajo del enfermero con base en la práctica clínica. Se trata de una investigación cualitativa y exploratoria, en forma de estudio de caso, realizada en una unidad de hospitalización quirúrgica. Se ha encuestado un grupo de seis enfermeros y los datos han sido recolectados a través de observación no participante y entrevista en profundidad. El periodo de recolección tuvo lugar entre octubre de 2012 y enero de 2013 y los datos han sido analizados por medio de análisis textual discursivo. Se ha verificado que la clínica, como campo de conocimiento, vuelve al trabajo de las enfermeras más visible con respecto a los diferentes individuos involucrados en el proceso de salud. Para ello, es necesario reconocer que este profesional incorpora la clínica a su trabajo. Siendo así, la clínica puede ser un instrumento que permite el reconocimiento y la consecuente visibilidad del trabajo del enfermero y contribuye para discusiones, negociaciones y transformaciones.

Palabras Clave: Competencia clínica; enfermería; trabajo; enfermería quirúrgica.
\end{abstract}

\section{INTRODUÇÃO}

O modelo clínico/biomédico constitui um marco importante nas organizações dos serviços de saúde, na assistência prestada e no próprio senso comum da populaçãoำ. E, nesse contexto, a atuação da enfermagem pode se limitar à sustentação das práticas médicas, constituindo-se como um trabalho complementar e pouco reconhecido 2 .

Contudo, esse modelo de atenção fundamenta-se em um arcabouço de conhecimentos indissociável da atuação profissional do enfermeiro: o conhecimento clínico - a clínica. Assim, apesar de haver uma contraposição entre um fazer integralizador e o fazer fragmentador do modelo clínico/biomédico, o enfermeiro pode encontrar, nesse modelo de atenção à saúde, meios que lhe permitam prestar um cuidado resolutivo.

Destaca-se, para tanto, o resgate da base científica nos cuidados ao paciente cirúrgico como aspecto importante no trabalho da enfermagem. Entretanto, os

IEnfermeira. Doutora em Enfermagem. Docente da Escola de Enfermagem da Universidade Federal. Rio Grande, Rio Grande do Sul, Brasil. Grupo de Estudos e Pesquisas em Organização do Trabalho da Enfermagem e Saúde. E-mail: lenicesousa@furg.br.

"Enfermeiro. Doutor em Enfermagem. Docente do Programa de Pós-Graduação em Enfermagem da Universidade Federal do Rio Grande. Rio Grande, Rio Grande do Sul, Brasil. Grupo de Estudos e Pesquisas em Organização do Trabalho da Enfermagem e Saúde. E-mail: vlunardi@terra.com.br.

IIIEnfermeira. Doutora em Enfermagem. Docente da Faculdade de Enfermagem e Obstetrícia da Universidade Federal de Pelotas. Pelotas, Rio Grande do Sul, Brasil.E-mail:mairabt@ufpel.tche.br. 
cuidados de enfermagem não podem se afastar das necessidades dos pacientes e devem ser avaliados quanto a sua qualidade e validade para cada cirurgia proposta ${ }^{3}$.

A produção do conhecimento em enfermagem no Brasil indica um distanciamento entre a Enfermagem e o conhecimento clínico. Esse afastamento fragiliza o campo teórico dos enfermeiros acerca da clínica como instrumental para a realização do seu trabalho ${ }^{4}$. Desse modo, pode ter havido um rompimento com alguns elementos da clínica que contribuem para sustentar a profissão e que constituem um dos fundamentos teóricos para o trabalho da enfermagem.

É necessário clarificar o saber construído e utilizado na prática clínica do enfermeiro, de forma que o reconhecimento profissional possa ser justificado pela ação concreta, para além das competências adquiridas na formação formal ${ }^{5}$. Desse modo, o presente estudo teve por objetivo analisar a visibilidade do trabalho do enfermeiro com base no exercício da clínica.

\section{Referencial TeÓRICO}

Neste estudo, a clínica é entendida como um campo no qual as práticas e saberes biomédicos e não biomédicos interagem, de modo a promover novas composições e modos de atuar em saúde ${ }^{6}$. Entende-se que para visualizar o indivíduo estabelecendo relações plenas com o ambiente e intervir sobre suas necessidades, é preciso atender aquelas que são indispensáveis para a manutenção da vida.

Para tanto, é necessário conhecimento clínico para sustentar o trabalho em enfermagem e buscar, por meio de uma visão ampliada, a integralidade da assistência. Assim, considera-se que quando a enfermagem se afasta da base científica que sustenta a clínica, ela fragiliza o seu saber e fazer, enfraquecendo as conexões que podem levar à integralidade do cuidado e à sua valorização como disciplina.

Entende-se que a resolutividade do trabalho do enfermeiro pode ocorrer mesmo que ele atue sob o modelo clínico/biomédico de assistência sem, no entanto, perder as características intrínsecas a um trabalho de enfermagem complexo e amplo. O reconhecimento do trabalho do enfermeiro reflete em sua visibilidade e, para tanto, torna-se importante que, ao desempenhar as práticas de cuidado, o enfermeiro demonstre conhecimento e competência técnica, associados à relação intersubjetiva e dialógica desenvolvida com a equipe, clientes, família e instituição?

Fundamentando-se nas ideias apresentadas, optou-se por utilizar, neste estudo, um referencial teórico-filosófico que permitisse aproximações conceituais capazes de provocar reflexões acerca da temática do estudo. A escolha por Deleuze e Guattari ocorreu por suas asserções a um pensar múltiplo e interconectado. Assim, são incorporados ao estudo os conceitos de linhas de fuga e máquina de guerra como forma de ancorar os achados a concepções que não delineassem limites à prática clínica do enfermeiro ${ }^{8}$.

\section{Metodologia}

Trata-se de uma pesquisa qualitativa, exploratória na forma de estudo de caso com coleta de dados executada entre outubro de 2012 e janeiro de 2013. Empregou-se o estudo de caso, pois este contribui com o conhecimento sobre os fenômenos individuais, organizacionais, sociais, políticos e de grupo, além de outros fenômenos relacionados ${ }^{9}$. Foi realizado em um setor de internação cirúrgica de um hospital universitário e teve como unidade de análise um grupo de seis enfermeiros do mesmo setor que foram identificados com letras e número em que $\mathrm{T}$ representa o turno e $\mathrm{P}$, o profissional.

A coleta de dados ocorreu por meio de observação não participante (200 horas) e entrevista em profundidade. As observações foram realizadas na unidade de internação cirúrgica, divididas em períodos de, pelo menos, quatro horas ininterruptas e de maneira semelhante entre os três turnos. Com base nas observações realizadas, emergiram as questões para as entrevistas em profundidade, a partir de uma análise inicial dos eventos observados.

Os dados foram analisados, de acordo com a análise textual discursiva. Tal método permite identificar e isolar enunciados dos conteúdos a ela submetidos, categorizá-los e produzir textos, de maneira a integrar descrição e interpretação ${ }^{10}$. Foram identificadas as unidades de análise do estudo, realizada a síntese das unidades constituintes mais similares e reintegração em categorias.

A execução da pesquisa foi submetida ao Comitê de Ética em Pesquisa na Área da Saúde e aprovada pelo parecer $n^{\circ} 87 / 2012$, tendo sido operacionalizada, segundo as diretrizes e normas da Resolução n466/2012 do Conselho Nacional de Saúde ${ }^{11}$.

\section{Resultados e Discussão}

O estudo permitiu observar que a resolutividade das necessidades dos pacientes, bem como uma concepção mais ampla de cuidado, resultam na visibilidade do trabalho do enfermeiro por diferentes sujeitos do processo de produção de saúde. Assim sendo, o produto da operacionalização desta pesquisa está organizado em dois momentos distintos, descritos a seguir.

\section{A resolutividade do trabalho em enfermagem sob o prisma das necessidades emergentes}

$O$ paciente de uma unidade de internação cirúrgica chega à instituição hospitalar com uma expectativa em especial: a realização de uma cirur- 
gia. Portanto, traz consigo expectativas quanto ao procedimento cirúrgico e aos cuidados empreendidos durante o perioperatório.

As necessidades de saúde são reconhecidas pelos pacientes, os quais requerem a resolução de seus problemas. $\mathrm{O}$ enfermeiro, ainda que pautado no modelo clínico, exerce papel relevante ao atender tais necessidades contidas nos aspectos biológicos.

Paciente com neoplasia de reto refere, durante o momento de visita profissional, que quer fazer logo a cirurgia para a resolução do problema e que isso é o que mais lhe incomoda no momento. (observação de T2P2)

A busca pela integralidade do cuidado não está somente em alcançar elementos que o modelo clínico não comporta. Ao questionar o modelo clínico quanto às suas limitações, por não contemplar a integralidade do cuidar, não se pretende rejeitá-lo e substituí-lo. Ao contrário, espera-se valorizar seus pontos positivos por meio de reconstruções teóricas e técnicas a serem incorporadas para que o olhar sobre o cuidado adquira uma competência política, social e cultural que permita uma identificação mais contextualizada e uma aplicação de condutas mais resolutivas ${ }^{6}$.

A integralidade traz, de maneira intrínseca, o comprometimento com a resolutividade daquelas necessidades prementes de cuidado. Nesse sentido, a avaliação clínica do enfermeiro pode subsidiar o cuidado ao paciente, de forma resolutiva, por meio dos problemas identificados e, ainda, nortear as condutas de outros profissionais da equipe de saúde, oferecendo meios para melhor cuidar.

O profissional, ao avaliar os pacientes, verifica drenagem em ferida operatória e analisa a possibilidade de abertura de pontos; assim, solicita a presença do médico residente para discutir a conduta a ser tomada. $\mathrm{O}$ médico residente realiza o procedimento nos pacientes indicados pela enfermeira. (observação de T2P1)

A clínica comporta conhecimentos que, incorporados pelo enfermeiro, lhe conferem um saber sobre as ações que devem ser empreendidas para a resolução das necessidades e determinar até onde suas condutas podem ser resolutivas ou não.

Uma técnica de enfermagem informa à enfermeira que um paciente permanece com febre e também tem apresentado alterações nos níveis de glicemia; a enfermeira menciona: 'Ele vai continuar assim, enquanto não for resolvido o problema. Enquanto não fizerem a amputação, ele vai continuar séptico e com alterações na glicemia, na pressão arterial, na respiração...'. (observação de T1P1)

O conhecimento clínico do enfermeiro interfere no processo terapêutico, quando ele implementa intervenções de cuidado ou mesmo quando encaminha o paciente para que outros profissionais o façam. Para tanto, o enfermeiro cria conexões com os demais membros da equipe de saúde, ou seja, estabelece linhas de fuga capazes de atingir os objetivos propostos e contribuir para a integralidade do cuidado. Desse modo, essas linhas de fuga são interconexões que, sustentadas pelo conhecimento clínico, atuam como meios de cuidar em saúde; promovem multiplicidades de transformação que a estruturam e criam novos caminhos ${ }^{8}$.

Contudo, a efetividade dessas inter-relações requer o estabelecimento de conexões e, diante da necessidade de intervenção de outros profissionais, o enfermeiro encontra, no reconhecimento e valoração de sua avaliação clínica, a conexão necessária para tal. Além disso, o reconhecimento do trabalho desempenhado pelos enfermeiros estimula e mobiliza o profissional, incentivando o seu fazer ${ }^{12}$.

Ao reconhecer que o enfermeiro tem conhecimento clínico e que sua atuação pauta-se na resolutividade de necessidades concretas, sabendo priorizar suas ações e estabelecer relações multiprofissionais, quando requisitados, os outros profissionais passam a considerar o parecer do enfermeiro.

Se estou precisando de alguém com urgência... Eu não quero para hoje. Eu quero para ontem. [...] Nem todo mundo tem isso, de se impor, na hora que necessita. [...] Se eu vejo que a coisa está ficando feia, eu ou qualquer outra enfermeira... Com o tempo, a gente já conseguiu respeito. Quando tu precisas, mas não existe um médico específico, se chamar, qualquer um vem aqui [...] (entrevista de T1P1)

Comunicar ao médico uma situação de urgência e solicitar sua presença imediata requer do enfermeiro o conhecimento necessário para identificar que sua atuação, isoladamente, não mais comporta as necessidades do paciente. Contrariamente, se o enfermeiro não sabe identificar uma ocorrência de emergência entre aquelas que requerem intervenções imediatas, esse profissional fragiliza as ligações de suas linhas de fuga.

A produção nacional sobre o trabalho de enfermagem está posta diante de importantes desafios como a consolidação das temáticas relacionadas à intersubjetividade no trabalho ${ }^{13}$. Assim, essas relações intersubjetivas na esfera de trabalho são pouco exploradas no que se refere à produção de conhecimento e, portanto, esses resultados ainda requerem mais discussões no meio científico.

Saber conduzir o estabelecimento das linhas de fuga para as inter-relações com outros profissionais tem importância no reconhecimento de seu saber/fazer. Além disso, o estabelecimento de prioridades e a identificação das alterações clínicas mais importantes podem refletir em uma prática clínica resolutiva e reconhecida.

Entretanto, por vezes, a resolutividade do trabalho do enfermeiro pode encontrar limitações na postura de outros profissionais ou na própria organização do trabalho da instituição. Tal aspecto pode demonstrar uma concepção de que a clínica é campo de apropriação médica e, portanto, não é instrumento fundamental para o trabalho em enfermagem. 
$\mathrm{Na}$ passagem de plantão, um enfermeiro informa ao outro que houve uma internação com diagnóstico de abdômen agudo. Indica que há suspeita de apendicite, mas o paciente não apresenta febre. Ainda, acrescenta que os exames laboratoriais não foram possivieis de serem avaliados porque estavam em posse da médica residente. (observação de T2P1 e T3P1)

A prática profissional médica alheia à equipe multiprofissional pode ser reflexo da própria organização do trabalho na instituição, que não estabelece normatizações às informações que devem ser disponibilizadas no prontuário do paciente. Estudo realizado com enfermeiros da atenção básica de São Paulo encontrou achados semelhantes que indicam um desconforto por parte dos enfermeiros diante do não reconhecimento do trabalho clínico do enfermeiro no âmbito da organização e gestão ${ }^{14}$.

Nesse cenário de atuação, a competência clínica do enfermeiro pode ser potencializada, por meio de formação contínua; entretanto, não se tem conhecimento de quais são as expectativas dos outros profissionais e da administração dos serviços de saúde quanto a isso. Nesse sentido, a história de cada categoria profissional pode intervir nesse processo, assim como a gestão dos serviços de saúde pode não compreender a importância da multiprofissionalidade e permanecer centrada na figura do médico ${ }^{5}$.

\section{A visibilidade do trabalho do enfermeiro como mecanismo fortalecedor na produção de saúde}

A visibilidade profissional do enfermeiro manifesta-se por meio do seu conhecimento científico, da sua participação na tomada de decisões referentes ao cuidado do paciente e/ou nas atividades de gerenciamento ${ }^{7}$. Desse modo, o conhecimento clínico pode ser uma ferramenta que possibilita a visibilidade do trabalho do enfermeiro e contribui nas discussões, negociações e transformações nos cenários de saúde.

Assim, para tornar o trabalho do enfermeiro visível, é necessário criar linhas de fuga que, de acordo com Deleuze e Guattari, compõem um mapa. Conforme esses autores, fazer um mapa é diferente de fazer um decalque, pois este provém de uma simples reprodução ${ }^{8}$.

trabalho em enfermagem não precisa reproduzir o modelo clínico, ele deve construir por meio de um mapa a busca pela integralidade. São as características do mapa, aberto, coletável em todas as suas dimensões, desmontável, reversível, suscetível de receber modificações que subsidiam um cuidado individualizado e resolutivo ${ }^{8}$.

Os enfermeiros reconhecem que a clínica, como campo de conhecimento, torna seu trabalho mais visível para os diferentes sujeitos envolvidos no processo de saúde.
O conhecimento clínico torna o trabalho mais visível para o enfermeiro, para os colegas de trabalho, para os técnicos de enfermagem que passam a te respeitar mais e o médico residente também passa a te respeitar mais. (entrevista de T2P2)

O domínio do conhecimento é condição que sustenta o desenvolvimento do papel clínico do enfermeiro e, deste modo, essencial para as intervenções de enfermagem ${ }^{15}$. Assim, o conhecimento sustenta a prática profissional e resulta em benefício ao paciente que, dessa forma, o reconhece. De maneira semelhante, os outros profissionais também podem reconhecer seu trabalho, ao perceberem que as ações empreendidas são resolutivas e transmitem cientificidade.

A forma de organização das profissões, bem como do trabalho, historicamente, foi modelado sob um enfoque fragmentado. Entretanto, há que se obter um trabalho mais integrador, pois existe a necessidade de vários olhares, inclusive para atender ao que preconiza o Sistema Único de Saúde, ao apontar a integralidade como um dos pilares de sustentação da atenção à saúde ${ }^{16}$.

Então, como tornar o trabalho mais visível? Entende-se que isso pode ocorrer por meio de atividades cotidianas individuais do enfermeiro, que se ampliam ao coletivo e refletem no trabalho em enfermagem de forma mais extensa.

O enfermeiro questiona ao médico residente da traumatologia se o raio-x de um paciente indica osteomielite; diagnóstico que esse profissional disse ter suspeitado, após avaliação da ferida do paciente. O residente confirma o diagnóstico de osteomielite. (observação de T2P1)

Dois enfermeiros solicitam avaliação médica de um paciente. Após a avaliação, os médicos são questionados, por um deles, acerca da conduta a ser tomada e diagnóstico identificado. Permanece no posto de enfermagem a discussão clínica acerca do caso. Uma terceira enfermeira chega ao posto de enfermagem para a passagem de plantão e recebe as informações sem fazer questionamentos. No posto de enfermagem, os médicos novamente voltam a fazer a discussão clínica por, pelo menos, 30 minutos. T3P2 permanece, durante todo o período, alheia à discussão, não mantendo qualquer comunicação com a equipe médica. (observação de T2P1, T2P2, T3P2)

A construção do papel clínico do enfermeiro requer participação ativa, para que, assim, possa assumir a autoria no seu desempenho, atribuindo particularidade às interações desenvolvidas com o paciente e com os demais agentes de saúde. Deste modo, ainda que o contexto seja favorável ao desempenho do papel clínico, é crucial a interação do enfermeiro para sua construção ${ }^{15}$.

A postura do enfermeiro pode tornar seu conhecimento mais ou menos visível na relação com os demais profissionais. As atitudes individuais contribuem para construir o status profissional do enfermeiro e, 
por sua vez, refletem-se na ampliação das intervenções sociais, mais expressivamente, na ocupação de espaços que deem margem e reconhecimento à enfermagem ${ }^{1}$.

Observa-se que o enfermeiro também pode tornar seu trabalho mais visível quando explicita os dados derivados de sua avaliação clínica. Desse modo, quando o enfermeiro fornece determinadas informações, ele realiza um refinamento, indica o que é mais relevante para cada caso, evidenciando conhecimento dos aspectos mais relevantes.

Um enfermeiro solicita avaliação da residente para um paciente com gangrena na perna, pois suspeita de sepse; informa à médica residente sinais e sintomas do paciente e salienta elevação de temperatura corporal, referindo tal suspeita. A médica foi realizar a avaliação e acredita que o paciente realmente está séptico. (observação de T1P1)

Ao estabelecer com a equipe médica linhas de fuga concretas que estabelecem um fluxo de interação em benefício do paciente, o enfermeiro, da mesma forma, colabora para que o contexto do cenário de saúde seja favorável à multiprofissionalidade. Entretanto, nem todos os outros profissionais da área de saúde se percebem autônomos para, assim como o enfermeiro, estabelecer linhas de fuga com os médicos.

Pode-se observar que, diante da necessidade de interação com o médico, os demais profissionais criam linhas de fuga com o enfermeiro para que este, então, as estabeleça com a equipe médica.

A fisioterapeuta informa à enfermeira que um paciente está com máscara de venturi, saturando bem, mas taquipneico e que ela julga necessário elevar a concentração de oxigênio. A enfermeira diz concordar e explica que o paciente está assim porque está séptico. Orienta que a fisioterapeuta telefone para a médica e discuta com ela a conduta. A enfermeira: 'Ela [fisioterapeuta] vem falar para mim a respeito de coisas que não são da minha conduta. Eu concordo com ela, mas, quando eu tenho que falar com os médicos, eu mesma falo... Eu não entendo porque ela vem falar para mim... Ela quer que eu fale com eles. Essa guria é boa, mas ela fala as coisas só para mim'. (observação de T1P1)

Ao reconhecer, no trabalho do enfermeiro, o conhecimento clínico necessário que lhe confere relações transversais na equipe multiprofissional, alguns profissionais da área da saúde podem atribuir ao enfermeiro uma referência para criação de linhas de fuga. Existem evidências de que o enfermeiro vem sendo reconhecido pelos profissionais da saúde como um articulador nos serviços de saúde, porque seu conhecimento envolve o contexto social mais amplo².

A clínica pode ainda ser percebida por alguns pacientes como campo de domínio médico e, portanto, o enfermeiro pode ser confundido com esse profissional, quando tem seu trabalho reconhecido e valorizado.
Uma familiar refere estar sendo muito bem atendida na instituição e que gostaria que tivesse um meio pelo qual pudesse deixar registrada sua opinião. Elogia o trabalho da enfermeira e a chama de doutora. Ela esclarece que é enfermeira e a familiar, então, conclui: 'Para mim, é doutora'. (observação de T2P2)

O estudo da história da enfermagem leva a compreender que os embates contra os preconceitos impostos pelo imaginário coletivo, através do conhecimento, têm importância na aceitação e reconhecimento do enfermeiro. Entretanto, na realidade profissional, frequentemente, o enfermeiro ainda pode ser confundido com outros profissionais ${ }^{17}$.

$O$ fato de o enfermeiro ser confundido denota um não reconhecimento de seu saber e fazer como enfermeiro. Desse modo, ainda que o conhecimento seja reconhecido e visível para alguns pacientes, os mitos construídos, no decorrer da história, podem invisibilizar a sua imagem como profissional detentor do saber clínico nos espaços de produção de cuidado.

Os pacientes podem reconhecer que o enfermeiro emprega, no seu trabalho, um corpo clínico de conhecimentos. Entretanto, talvez, o desafio seja torná-lo visível com contornos coletivos. A visibilidade do trabalho tem que extrapolar os limites individuais e colaborar para a visibilidade da figura do enfermeiro como profissional autônomo e clinicamente competente.

Nesse sentido, o enfermeiro é seu próprio outdoor e, portanto, trabalhar sua postura humana é importante para a visibilidade de seu conhecimento. Contudo, salienta-se que é preciso ter cuidado para que a construção da imagem profissional seja coerente com o seu saber e não somente constituída na aparência de um bom profissional ${ }^{17}$.

A clínica pode configurar-se como uma máquina de guerra, atuando no sentido de fortalecer a imagem do enfermeiro como profissional competente e qualificado clinicamente. Uma máquina de guerra corresponde ao resultado de processos de subjetivação que criam uma máquina abstrata, um meio de atuar contra o estabelecido, um modo de o enfermeiro prestar cuidados que, confronta o modelo instituído sem, no entanto, negá-lo ${ }^{8}$.

\section{Conclusão}

Fundamentando-se no referencial filosófico de Deleuze e Guattari, verificou-se que, na prática clínica, o enfermeiro direciona suas ações para o cuidado com o paciente ou cria linhas de fuga com os demais membros da equipe de saúde, contribuindo para a integralidade do cuidado. Sob esse prisma, a clínica, como campo de conhecimentos, torna o trabalho do enfermeiro mais visível para os diferentes sujeitos envolvidos no processo de saúde. 
A pesquisa apresenta limitações, por se tratar de um estudo de caso, ainda que desenvolvido com um grupo de enfermeiros, em um local específico de aplicação. Entretanto, permitiu identificar que o enfermeiro pode ser o profissional tido como referência para a criação das linhas de fuga. Para tanto, é necessário o reconhecimento de que esse profissional incorpora o conhecimento clínico para o estabelecimento de articulações e relações transversais na equipe multiprofissional. Assim, a clínica pode contribuir nas discussões, negociações e transformações nos cenários de saúde, colaborando para um agir multiprofissional mais efetivo.

Ainda que a atuação do enfermeiro ocorra no modelo clínico/biomédico de assistência, esse profissional busca, no exercício da clínica, meios para um cuidar em saúde mais amplo e, da mesma forma, o utiliza como instrumento para a resolutividade e visibilidade do trabalho em enfermagem. Deste modo, a clínica pode configurar-se como uma máquina de guerra criada a partir das linhas de fuga - uma força em potencial -, contrapondo-se a modelos hierarquizados e atuando no sentido de fortalecer a imagem do enfermeiro como profissional competente e qualificado clinicamente.

\section{REFERÊNCIAS}

1.Dal Pai D, Schrank G, Pedro ENR. O enfermeiro como ser sócio-político: refletindo a visibilidade da profissão do cuidado. Acta Paul Enferm. 2006; 19: 82-7.

2.Erdmann AL, Fernandes JV, Melo C, Carvalho BR, Menezes Q, Freitas R, et al. A visibilidade da profissão de enfermeiro: reconhecendo conquistas e lacunas. Rev Bras Enferm. 2009; 62: 637-43.

3.Christoforo BEB, Carvalho DS. Cuidados de enfermagem realizados ao paciente cirúrgico no período pré-operatório. Rev esc enferm USP. 2009; 43: 14-22.

4.Sousa LD, Lunardi Filho WD, Lunardi VL, Santos SS, Santos CP. A produção científica de enfermagem acerca da clínica: uma revisão integrativa. Rev esc enferm USP. 2011; 45: 494-500.

5.Basto ML. Da (in) visibilidade do trabalho das enfermeiras à produção de saberes em enfermagem: cuidados prestados num centro de saúde. Revista Portuguesa de Saúde Pública. 2005; 23: 25-41.

6.Favoreto C. A prática clínica e o desenvolvimento do cuidado integral à saúde no contexto da atenção primária. Revista de APS. 2008; 11: 100-8.

7.Castanha ML, Zagonel IPS. A prática de cuidar do ser enfermeiro sob o olhar da equipe de saúde. Rev Bras Enferm. 2005; 58: 556-62.

8.Deleuze G, Guattari F. Mil Platôs: capitalismo e esquizofrenia. v.5. São Paulo: Editora 34; 2000.

9.Yin RK. Estudo de caso: planejamento e métodos. Porto Alegre (RS): Bookman; 2005.

10.Moraes R, Galiazzi MC. Análise textual discursiva. Ijuí (RS): Unijuí; 2007.

11.Ministério da Saúde (Br). Conselho Nacional de Saúde. Resolução no 466/2012. Dispõe sobre pesquisa envolvendo seres humanos. Brasília (DF): CNS; 2012. 12.Sprandel LIS, Vaghetti HH. Valorização e motivação de enfermeiros na perspectiva da humanização do trabalho nos hospitais. Rev Eletr Enf. [Internet]. 2012; 14: 794-802.

13.Mandú ENT, Peduzzi M, Silva AMN. Análise da produção científica nacional sobre o trabalho de enfermagem. Rev enferm UERJ. 2012; 20: 118-23.

14. Matumoto S, Fortuna CM, Kawata LS. A prática clínica do enfermeiro na atenção básica: um processo em construção. Rev Latino-Am Enfermagem. 2011; 19(1): [08 telas].

15.Mendes MA. Papel clínico do enfermeiro: desenvolvimento do conceito. [tese publicada]. São Paulo: Universidade de São Paulo; 2010.

16.Gelbcke FL, Matos E, Sallum NC. Desafios para a integração multiprofissional e interdisciplinar. Revista Tempus Actas de Saúde Coletiva. 2012; 6: 31-9.

17.Gentil R. C. O enfermeiro não faz marketing pessoal: a história explica por quê? Rev Bras Enferm. 2009; 62: 916-8. 